\title{
Estudo comparativo de dois eletrodos associados ao plasma elétrico de alta frequência na redução do crescimento de cepas clínicas de Malassezia spp.
}

\author{
Comparative study of two electrodes associated to high frequency electric plasma in the \\ reduction of the growth of clinical strains of Malassezia spp.
}

\author{
Ana Beatriz Furtado Rodrigues ${ }^{1}$, Raissa Albano da Silva ${ }^{2}$, \\ Raduan $\mathrm{Hage}^{3}$, Sônia Khouri4.
}

\section{RESUMO}

Introdução: Malassezia spp. é um gênero leveduriforme, comumente isolado da microbiota normal do conduto auditivo de cães, que possui espécies associadas a otites externas e dermatites atópicas. O plasma elétrico é considerado o quarto estado da matéria, que vem sendo empregado na descontaminação e esterilização. Objetivo: Avaliar os efeitos do plasma elétrico de alta frequência, utilizando dois diferentes eletrodos de vidro, na redução do crescimento de cepas clínicas de leveduras do gênero Malassezia spp. Material e Métodos: Nove amostras de Malassezia spp., isoladas do conduto auditivo de cães com otite externa, foram tratadas durante 6 minutos com plasma elétrico de alta frequência por meio de dois diferentes eletrodos de vidro. Após 72 horas de incubação em estufa, as amostras foram analisadas e comparadas com amostras controle. Resultados: Verificou-se que nas amostras tratadas com plasma de alta frequência, por meio do eletrodo esférico maior, houve uma redução considerável no número de unidades formadoras de colônias, cerca de $82 \%$, em relação ao controle positivo. Conclusão: O plasma de alta frequência mostrou-se como uma técnica promissora no controle in vitro de cepas clínicas de Malassezia spp. induzindo um percentual médio de redução de $82 \%$ quando tratadas com o eletrodo do tipo esférico, o mesmo não foi observado quando utilizado o eletrodo do tipo cauterizador que apresentou um percentual de redução médio de apenas 32\%.

Descritores: Malassezia spp. Cães. Otite externa. Gases de plasma. Plasma a frio.

\section{ABSTRACT}

Introduction: Malassezia spp. is a yeast gene, commonly abnormal of the microbiota of the auditory canal of dogs, which has species associated to external otitis and atopic dermatitis. The electric plasma is considered the fourth state of matter, which has been used in decontamination and sterilization. Aim: to evaluate the effects of high frequency electric plasma, using two different dosages of glass, in reducing the growth of clinical strains of yeasts of the genus Malassezia spp. Material and Methods: Nine samples of Malassezia spp. isolated from the auditory canal of dogs with external otitis were treated for 6 minutes with high frequency electric plasma through two different glass electrodes. After 72 hours of incubation in the greenhouse, the samples were analyzed and compared with control. Results: It was verified that in the tests treated with high frequency plasma, by means of the larger spherical electrode, there was a considerable reduction in the number of colony forming units, about $82 \%$, in comparison to the positive control. Conclusion: High-frequency plasma showed to be a promising non-invasive technique of clinical strains of Malassezia spp. The average percentage of reduction of $82 \%$ when treated with the spherical type, the same was not done when using the type of control cauterizador that reduced the average percentage of only $32 \%$.
'Discente do curso Biomedicina,

Universidade do Vale do

Paraíba/UNIVAP.

E-mail:

beatrizrodrigues.abfr@gmail.com

2Discente do curso de

Biomedicina, Universidade do

Vale do Paraíba/UNIVAP.

3Médico Veterinário, doutor,

docente na Universidade do Vale

do Paraíba, Universidade do Vale

do Paraíba/UNIVAP

${ }^{4}$ Biomédica, doutora, docente e coordenadora do curso de Biomedicina da Universidade do Vale do Paraíba, Universidade do Vale do Paraíba/UNIVAP.

Keywords: Malassezia spp. Dogs. Cold plasma. Plasma gases 
Rodrigues ABF. Silva RA. Hage R. Khouri S.

Estudo comparativo de dois eletrodos associados ao plasma elétrico de alta frequência na redução do crescimento de cepas clínicas de Malassezia spp.

\section{INTRODUÇAOO}

Malassezia spp. é um gênero de levedura, do filo Basidiomiceto, que compreende, de acordo com estudos recentes, cerca de 15 espécies lipofílicas, sendo a maioria delas lipidiodependentes e apenas uma não lipidiodependente. Este fungo é comumente isolado da microbiota normal da pele, conduto auditivo e mucosas de cães, mas também pode ser encontrado na microbiota de seres humanos, aves e outros mamíferos. Esta levedura apresenta espessa parede celular, formada por quitina e lipídeos em abundância. Este último fator auxilia na proteção contra a atividade de antifúngicos, dificultando seu tratamento e controle. Contudo, a patogenicidade desta levedura pode estar relacionada à características do hospedeiro, como desordens hormonais ou imunológicas, que favorecem seu crescimento como oportunista. ${ }^{1-6}$

Diversas espécies de Malassezia spp. foram associadas as infecções cutâneas e as otites externas e dermatites atópicas em cães. Conforme dados da literatura, as otites eternas provocadas por espécies do gênero Malassezia podem ser observadas em cães jovens e sadios, sendo Malassezia furfur e Malassezia pachydermatis as principais espécies isoladas na prática veterinária. As otites podem se manifestar em decorrência de causas primárias, como alergias alimentares e sistema imunológico do hospedeiro deficiente, mas também quando ocorre alguma alteração no conduto auditivo, tais como mudanças de temperatura, $\mathrm{pH}$ ou umidade, permitindo que este micro-organismo deixe de viver como comensal e se torne um patógeno oportunista. 1,2,4,6,7

Segundo a física, o plasma é considerado o quarto estado da matéria e está presente em todo o universo. Ele pode ser encontrado como um gás ou vapor, ionizado ou energizado, que pode ser criado a partir de aplicação de energia, na forma de calor ou campo eletromagnético, descargas elétricas (como relâmpago) ou reações nucleares controladas. Para que o plasma seja gerado, é necessário haja uma diferença de potencial elétrico, que promova a excitação do gás, acelerando suas partículas. Logo, as moléculas de um gás (como hélio, oxigênio e argônio) depois de excitadas, perdem seus elétrons, gerando uma mistura de partículas carregadas positiva e negativamente, em igual proporção, íons negativos e positivos, entre outras espécies reativas. ${ }^{3,8-12}$

Há dois tipos de plasma: os de baixa temperatura, também chamados de nãotérmicos, e os de alta temperatura, ou térmicos. Os plasmas de alta temperatura são menos 
DOI:10.18606/2318-1419/amazonia.sci.health.v7n3p2-11 Revista Amazônia: Science \& Health

2019, Vol. 7, № 3

ISSN: 2318-1419
Rodrigues ABF. Silva RA. Hage R. Khouri S.

Estudo comparativo de dois eletrodos associados ao plasma elétrico de alta frequência na redução do crescimento de cepas clínicas de Malassezia spp.

usados devido à elevada temperatura que é preciso para produzi-los (cerca de $3000^{\circ} \mathrm{C}$ ). Por outro lado, os plasmas de baixa temperatura podem ser produzidos a partir de microondas e rádio frequência, quando gases são estimulados à pressão atmosférica ou à baixa pressão, como os gerados a vácuo. 8,10,13

O plasma vem sendo empregado na área industrial, por exemplo, na modificação de superfícies e, mais atualmente, na desinfecção e esterilização de materiais na área médica e dispositivos odontológicos. Entretanto, este uso ainda não é amplamente difundido. Por este motivo, seu uso no controle microbiano vem sendo mais pesquisado. Estudos mais recentes têm demonstrado que o plasma apresenta amplo espectro de ação sobre microorganismos, pois não possibilita a formação de resistência. Apesar disso, príons e esporos necessitam ser tratados por mais tempo se comparados aos demais micro-organismos quando se deseja eliminá-los. Sendo assim, o plasma pode servir como uma alternativa limpa e que não gera resíduos, mas também na redução e inativação microbiana. ${ }^{11-14}$

Contudo, os mecanismos de ação dos plasmas não estão totalmente elucidados. Sabe-se que o gás, depois de ionizado, tem seus átomos e moléculas fragmentados, produzindo espécies reativas, íons e elétrons. Logo, sabe-se que as espécies reativas reagem com a membrana das células, mas também com moléculas em sua superfície e com ácidos nucleicos e proteínas internas das células. Sugere-se que a inativação dos micro-organismos pode ser decorrente de danos ao DNA, pela radiação UV ou pela atividade citotóxica de espécies reativas geradas pelo plasma. Pode ocorrer ainda modulação proteica e indução da apoptose. ${ }^{11,14,15}$

Um estudo realizado por Taghizadeh et al. ${ }^{3}$ cujo objetivo foi empregar o plasma nãotérmico na inativação de biofilmes de três diferentes micro-organismos (Candida albicans, Pseudomonas aeruginosa e Staphylococcus aureus) demonstrou que ele foi capaz de reduzir o crescimento microbiano. Isto indica que o plasma apresentou efetiva atividade antimicrobiana, assim como outros trabalhos também evidenciaram. Ademais, um estudo realizado por Ouf, Basher e Mohamed (2014), empregou plasma no controle de esporos do fungo Aspergillus niger em frutas, e mostrou que ele promoveu a redução destes esporos, sendo necessário, porém, maior tempo de exposição dos micro-organismos ao plasma, conforme outros estudos também já sugeriram. Por este motivo, a eliminação de microorganismos depende não apenas do tempo de exposição da amostra ao plasma, mas também da distância da mesma em relação ao jato e da intensidade aplicada. 11,12,14,16 
Rodrigues ABF. Silva RA. Hage R. Khouri S.

Estudo comparativo de dois eletrodos associados ao plasma elétrico de alta frequência na redução do crescimento de cepas clínicas de Malassezia spp.

O plasma pode ser produzido a partir de um gerador de alta frequência. Este gerador é um equipamento que fornecerá uma corrente de alta frequência a partir de uma corrente elétrica que, unido a um eletrodo de vidro que contém um gás, levará a formação do plasma. $3,9,12$

Um dos gases mais empregados na formação do plasma é o ozônio. Este gás vem sendo empregado em diversas situações, como o tratamento de feridas, graças a sua atividade oxidativa, que pode ser útil na esterilização e descontaminação, mas também na redução e inibição do crescimento de micro-organismos in vitro ou in vivo. Isto se dá, segundo estudos recentes propõem, pelo fato de que o ozônio pode comprometer a permeabilidade celular e oxidar aminoácidos e ácidos nucleicos. 3,10,12

O objetivo do presente estudo foi comparar os efeitos do plasma elétrico de alta frequência, utilizando-se dois tipos de eletrodos, esférico e tipo cauterizador, na redução do crescimento de cepas clínicas de Malassezia spp. isoladas do conduto auditivo externo de cães com sinais clínicos de otite externa.

\section{MATERIAIS E METODOS}

O presente estudo se trata de um estudo experimental, realizado entre os meses de agosto e novembro de 2018, no Núcleo de Estudos Farmacêuticos e Biomédicos (NUFABI) da Universidade do Vale do Paraíba (UNIVAP).

Inicialmente, foram colhidas 18 amostras do conduto auditivo externo de cães com sinais clínicos de otite externa, atendidos em um hospital veterinário, no serviço de dermatologia, na cidade de São José dos Campos, por uma médica veterinária dermatologista. As amostras foram colhidas com swabs secos estéreis que depois foram passados em placas de Petri com ágar Sabouraud com cloranfenicol a 0,05\%. Estas placas foram levadas ao NUFABI, onde foram incubadas em estufa a $35^{\circ} \mathrm{C}\left( \pm 2^{\circ} \mathrm{C}\right)$, por 72 horas.

Das 18 amostras colhidas, 09 foram confirmadas com coloração de Gram como positivas para Malassezia spp., e empregadas no estudo. As demais amostras foram descartadas devido à presença de contaminantes e à contagem inferior a 25 exemplares de Malassezia spp. observadas ao microscópio. Depois de isoladas e cultivadas, iniciaramse os testes, realizados em duplicata, com os dois eletrodos de vidro. Para isto, foram preparados inóculos das amostras de Malassezia spp., cultivadas 72 horas antes de cada experimento. Os inóculos foram preparados em solução salina $0,9 \%$, seguindo a escala de 
Rodrigues ABF. Silva RA. Hage R. Khouri S.

Estudo comparativo de dois eletrodos associados ao plasma elétrico de alta frequência na redução do crescimento de cepas clínicas de Malassezia spp.

turvação 0,5 de Mac Farland. Então, os inóculos foram diluídos até 1:100, em eppendorfs estéreis, com volume total de $1 \mathrm{~mL}$. Deste inóculo, $120 \mu \mathrm{L}$ foram pipetados em um poço de uma placa de Kline, que foi disposta a $1 \mathrm{~cm}$ de distância do eletrodo, exposto ao plasma elétrico de alta frequência por 6 minutos, tempo esse determinado em estudo piloto. Logo após, $100 \mu \mathrm{L}$ da solução tratada foram pipetados em uma placa de ágar Sabouraud com cloranfenicol a $0,05 \%$ e colocadas em estufa a $35^{\circ} \mathrm{C}$, por 72 horas.

Com o intuito de analisar se o tipo de eletrodo utilizado poderia interferir nos efeitos do plasma de alta frequência sobre a formação de UFCs, dois diferentes eletrodos foram utilizados e assim foram criados diferentes grupos no presente estudo: Grupo I - tratado com o eletrodo de vidro do tipo esférico maior; Grupo II - tratado com o eletrodo de vidro do tipo cauterizador; Grupo controle positivo - contendo apenas o inóculo de Malassezia spp. sem qualquer tratamento; Grupo controle negativo: contendo o inóculo de Malassezia spp. associado ao nitrato de Miconazol (0,02 g/mL).

Transcorrido o tempo de incubação de 72 horas, as placas foram retiradas da estufa, foi feita a contagem de UFCs/mL e determinação do percentual de redução de cada amostra e os resultados foram tabulados.

Este estudo foi submetido ao Comitê de Ética e Pesquisa de Animais da Universidade Paulista, sob o protocolo de aprovação no 055/16.

\section{RESULTADOS}

Os resultados obtidos após a contagem de unidades formadoras de colônias (UFCs/mL) dos grupos tratados (Grupos I e II) e dos grupos controle (positivo e negativo) encontram-se dispostos na tabela 1. Nota-se que houve redução no número de UFCs/ml em ambos os grupos tratados com plasma elétrico de alta frequência, porém no grupo I, que utilizou o eletrodo esférico maior, a redução foi muito maior em relação àquele que utilizou o eletrodo do tipo cauterizador. 
DOI:10.18606/2318-1419/amazonia.sci.health.v7n3p2-11 Revista Amazônia: Science \& Health

2019, Vol. 7, № 3

ISSN: $2318-1419$
Rodrigues ABF. Silva RA. Hage R. Khouri S.

Estudo comparativo de dois eletrodos associados ao plasma elétrico de alta frequência na redução do crescimento de cepas clínicas de Malassezia spp.

Tabela 1. Valores médios de UFCs $/ \mathrm{mL}$ e respectivos percentuais de redução dos diferentes grupos de amostras. O grupo controle negativo (Malassezia spp. + miconazol) não foi inserido na tabela pois não houve formação de UFCs em nenhuma das amostras.

\begin{tabular}{lcccccc} 
Amostras & \multicolumn{3}{c}{ Grupo I } & \multicolumn{3}{c}{ Grupo II } \\
\cline { 2 - 7 } & \multicolumn{2}{c}{ (eletrodo do tipo esférico) } & \multicolumn{2}{c}{ (eletrodo do tipo cauterizador) } \\
\cline { 2 - 8 } & $\begin{array}{c}\text { Controle } \\
\text { positivo }\end{array}$ & Teste & $\%$ redução & $\begin{array}{c}\text { Controle } \\
\text { positivo }\end{array}$ & Teste & $\%$ redução \\
& $5,40 \times 10^{4}$ & $1,40 \times 10^{3}$ & $97 \%$ & $1,38 \times 10^{6}$ & $8,55 \times 10^{5}$ & $38 \%$ \\
& $1,48 \times 10^{7}$ & $4,92 \times 10^{6}$ & $67 \%$ & $1,62 \times 10^{6}$ & $1,50 \times 10^{6}$ & $7 \%$ \\
2 & $5,10 \times 10^{4}$ & $2,20 \times 10^{4}$ & $57 \%$ & $2,40 \times 10^{5}$ & $1,70 \times 10^{5}$ & $29 \%$ \\
3 & $4,60 \times 10^{4}$ & $9,00 \times 10^{3}$ & $80 \%$ & $1,15 \times 10^{5}$ & $4,0 \times 10^{4}$ & $65 \%$ \\
5 & $3,35 \times 10^{5}$ & $5,00 \times 10^{3}$ & $98 \%$ & $2,75 \times 10^{5}$ & $1,85 \times 10^{5}$ & $33 \%$ \\
6 & $8,30 \times 10^{4}$ & $1,00 \times 10^{3}$ & $99 \%$ & $7,05 \times 10^{5}$ & $6,60 \times 10^{5}$ & $74 \%$ \\
7 & $5,24 \times 10^{6}$ & $1,72 \times 10^{6}$ & $67 \%$ & $1,48 \times 10^{6}$ & $1,02 \times 10^{6}$ & $31 \%$ \\
8 & $2,00 \times 10^{4}$ & 0 & $100 \%$ & $6,80 \times 10^{5}$ & $5,70 \times 10^{5}$ & $16 \%$ \\
9 & $2,50 \times 10^{4}$ & $6,00 \times 10^{3}$ & $76 \%$ & $5,20 \times 10^{5}$ & $2,70 \times 10^{5}$ & $48 \%$ \\
\hline
\end{tabular}

\section{DISCUSSAO}

Atualmente, sabe-se que a gama de antifúngicos convencionais é pequena e que os fungos têm apresentado crescente aumento de resistência ao uso dos mesmos. Além disso, os tratamentos disponíveis muitas vezes geram efeitos colaterais. Visto isso, há necessidade de se encontrar novos métodos seguros e confiáveis, que possam ser empregados nos tratamentos de infecções fúngicas.

A partir dos resultados obtidos no presente estudo, verificou-se que o plasma elétrico de alta frequência apresentou efeito inibitório sobre o crescimento de cepas clínicas de Malassezia spp. É possível notar ainda que o percentual de redução de UFCs $/ \mathrm{mL}$ foi maior naquelas amostras tratadas com o eletrodo do tipo esférico maior do que nas amostras tratadas com o eletrodo do tipo cauterizador. Tal diferença pode estar associada a maior superfície do eletrodo esférico levando a maior difusão do plasma nos inóculos, 
DOI:10.18606/2318-1419/amazonia.sci.health.v7n3p2-11

Revista Amazônia: Science \& Health

2019, Vol. 7, № 3

ISSN: 2318-1419
Rodrigues ABF. Silva RA. Hage R. Khouri S.

Estudo comparativo de dois eletrodos associados ao plasma elétrico de alta frequência na redução do crescimento de cepas clínicas de Malassezia spp.

consequentemente exercendo maior efeito inibitório sobre o crescimento dos microorganismos. Liao et al ${ }^{15}$ notaram que a eficiência do plasma a frio na inativação de microorganismos depende do tipo de plasma empregado, do tipo de superfície em que o microorganismos se encontra e do tipo de micro-organismo testado. Além disso, há fatores, como temperatura ambiente, matriz celular, $\mathrm{pH}$, tempo de exposição e características dos microorganismos que influenciam na ação do plasma. Essas observações corroboram com o presente estudo, no qual foi possível notar a marcante diferença de efeitos do plasma elétrico sobre o crescimento das cepas de Malassezia spp. alterando-se apenas o tipo de eletrodo utilizado, onde o eletrodo esférico promoveu um efeito muito mais intenso comparado ao tipo cauterizador, demonstrado por meio do percentual de redução de UFCs.

Annunziatta et al ${ }^{21}$ observaram que o plasma a frio foi eficaz na remoção de bactérias contaminantes de superfícies de titânio e, por este motivo, poderia ser uma alternativa eficiente para descontaminar e até mesmo esterilizar superfícies.

No estudo de Lee et al ${ }^{23}$ foram testadas cepas padrão de E. coli, S. Typhimurium e L. monocytogenes com plasma de gás nitrogênio e gás hélio misturado ao gás oxigênio, num tempo de tratamento de 10 minutos. Como resultado, foi visto que o plasma a frio foi capaz de inativar as cepas tratadas, havendo redução das cepas de S. Typhimurium de 1.6 para $1.5 \mathrm{log}$. Contudo, notaram que a inativação depende não somente do tempo de tratamento, mas também de fatores externos, como pH do meio. Deste modo e, com base nos resultados apresentados no presente trabalho, mais estudos são necessários empregando o plasma de alta frequência, com um tempo maior de irradiação, mas também com um número maior de amostras. Isto porque, para a realização do presente estudo foi realizado inicialmente um ensaio em que foram testados diferentes tempos de irradiação, contando com os seguintes tempos: 3, 6, 9 e 12 minutos, baseados em um estudo anterior feito com o mesmo plasma e as mesmas amostras e no qual observou-se que um tempo de tratamento inferior a 3 minutos não apresentava redução no número de UFCs.

Uma hipótese para justificar este tempo maior de exposição poderia estar no fato de que o presente estudo foi realizado com cepas clínicas. As cepas foram obtidas de diferentes cães e, por isso, podem apresentar diferentes características comportamentais e moleculares entre si. E, ao compararmos com cepas padrão, que são mais sensíveis, pode-se justificar a provável resistência ao tratamento empregado.

Como relatado anteriormente, pode-se notar que há variação na contagem de UFCs $/ \mathrm{mL}$ das amostras e que isto se deve ao fato de que foram utilizadas amostras clínicas, 
DOI:10.18606/2318-1419/amazonia.sci.health.v7n3p2-11 Revista Amazônia: Science \& Health

2019, Vol. 7, № 3

ISSN: 2318-1419
Rodrigues ABF. Silva RA. Hage R. Khouri S.

Estudo comparativo de dois eletrodos associados ao plasma elétrico de alta frequência na redução do crescimento de cepas clínicas de Malassezia spp.

colhidas de diferentes cães. Logo, a variação de crescimento, mas também de resistência mediante o tratamento, podem ser decorrentes de diferenças existentes dentro de um mesmo gênero.

Os trabalhos realizados por Ziuzina ${ }^{19,20}$ evidenciaram que o plasma a frio foi eficiente no controle de Salmonella Typhimurium, E. coli e L. monocytogenes isolados de tomate, tomate cereja, morango e alface. Nestes estudos foi observada inibição do crescimento de isolados de Salmonella Typhimurium obtidos de tomate, após 10 segundos de tratamento, e inibição do crescimento de isolados de alface após 30 segundos de tratamento. Foi verificado ainda que houve diminuição de $3.1 \log _{10}$ para $2 \log _{10}$ das amostras de $E$. coli após 45 segundos de tratamento com o plasma. Apesar da variação de tempo entre o estudo de Ziuzina $^{19,20}$ e o presente estudo, 10 segundos e 300 segundos (6 minutos), respectivamente, pode-se sugerir, com base na observação dos resultados, a grande capacidade inibitória do plasma de alta frequência no crescimento de diferentes tipos de micro-organismos.

\section{CONSIDERAÇOES FINAIS}

O plasma de alta frequência apresentou-se como uma técnica promissora no controle de cepas clínicas de Malassezia spp. in vitro, principalmente utilizando-se o eletrodo do tipo esférico, que cujo percentual de redução médio do número de UFCs/mL das cepas testadas foi de $82 \%$.

\section{REFERENCIAS}

1. Bandeira IB, Nunes AG, Vandesmet MLCS. Malassezia sp.revisión literaria sobre los aspectos generales. Unicatólica. 2016;1(88).

2. Böhmová E, Čonková E, Sihelská Z, Harčárová M. Diagnostics of Malassezia Species: A Review. Folia Vet [Internet]. 2018;62(2):19-29. Available from: http://content.sciendo.com/view/journals/fv/62/2/articlep19.xml

3. Eduardo C, Braz C, Solange P, Nunes RD, Denise S, Herrera SC, et al. Aplicação de aparelho de alta frequência e do vapor de ozônio no fungo malassezia spp Application of high-frequency equipment and steam ozone in the fungus malassezia spp. Rev Amaz Sci Heal. 2014;(63):29-34.

4. Brilhante RSN, Rocha MG da, Guedes GM de M, Oliveira JS de, Araújo G dos S, España JDA, et al. Malassezia pachydermatis from animals: Planktonic and biofilm antifungal susceptibility and its virulence arsenal. Vet Microbiol. 2018;220(May):47-52.

5. Carrillo-Muñoz AJ, Rojas F, Tur-Tur C, de los Ángeles Sosa M, Diez GO, Espada CM, et al. In vitro 
DOI:10.18606/2318-1419/amazonia.sci.health.v7n3p2-11 Revista Amazônia: Science \& Health

2019, Vol. 7, № 3

ISSN: 2318-1419
Rodrigues ABF. Silva RA. Hage R. Khouri S.

Estudo comparativo de dois eletrodos associados ao plasma elétrico de alta frequência na redução do crescimento de cepas clínicas de Malassezia spp.

antifungal activity of topical and systemic antifungal drugs against Malassezia species. Mycoses. 2013;56(5):571-5.

6. Celis AM, Vos AM, Triana S, Medina CA, Escobar N, Restrepo S, et al. Highly efficient transformation system for Malassezia furfur and Malassezia pachydermatis using Agrobacterium tumefaciens-mediated transformation. J Microbiol Methods [Internet]. 2017;134:1-6. Available from: http://dx.doi.org/10.1016/j.mimet.2017.01.001.

7. Almeida MDS, Santos SB, Mota ADR, Da Silva LTR, Silva LBG, Mota RA. Isolamento microbiológico do canal auditivo de cães saudáveis e com otite externa na região metropolitana de Recife, Pernambuco. Pesqui Vet Bras. 2016;36(1):29-32.

8. Afshari R, Hosseini $\mathrm{H}$. Non-thermal plasma as a new food preservation method, Its present and future prospect. J Paramed Sci Winter. 2014;5(1):2008-4978.

9. Bampi GM. AÇÃO BACTERICIDA (IN VITRO) DO GERADOR DE ALTA FREQUÊNCIA SOBRE CULTURA BACTERIANA COMUMENTE ENCONTRADA EM FERIDAS CRÔNICAS. Diss Univ Caxias do Sul, Programa PósGraduação em Biotecnol 2015 [Internet]. 2015 Mar; Available from: http://www.scielo.br/scielo.php?script=sci_arttext\&pid=S1676-06032011000100033\&lng=pt\&tlng=pt

10. Melo FL de. Efeitos Do Processamento Por Plasma Atmosférico Sobre As Características de Qualidade em Suco de Uva "Isabel". 2015.

11. Shintani H (Hideharu), Sakudo A. Gas plasma sterilization in microbiology : theory, applications, pitfalls and new perspectives [Internet]. [cited 2018 Nov 30]. 157 p. Available from: https://books.google.com.br/books?id=mXA7DwAAQBAJ\&pg=PA39\&lpg=PA39\&dq=shintani+sakudo\&so urce=bl\&ots=QruShfQ0LJ\&sig=XqZ_3xGemQiRYXTYQc_FYsKH-2E\&hl=pt-

BR\&sa=X\&ved=2ahUKEwjF7LDJ7PzeAhWJHZAKHYsuAvgQ6AEwAnoECAgQAQ\#v=onepage\&q=shinta ni sakudo\&f=false.

12. Wietzikoski Lovato EC, Gurgel Velasquez PA, dos Santos Oliveira C, Baruffi C, Anghinoni T, Machado RC, et al. High frequency equipment promotes antibacterial effects dependent on intensity and exposure time. Clin Cosmet Investig Dermatol. 2018;11:131-5.

13. Colombo LMPC, Matafora FL, Moro AFV. Condicionamento de superfícies na Odontologia com plasma de argônio: uma revisão de literatura. Rev Bras Odontol [Internet]. 2014;71(1):85-8. Available from: http://revodonto.bvsalud.org/scielo.php?script=sci_arttext\&pid=S0034-72722014000100018.

14. Ouf SA, Basher AH, Mohamed AAH. Inhibitory effect of double atmospheric pressure argon cold plasma on spores and mycotoxin production of Aspergillus niger contaminating date palm fruits. J Sci Food Agric. 2015;95(15):3204-10.

15. Liao X, Liu D, Xiang Q, Ahn J, Chen S, Ye X, et al. Inactivation mechanisms of non-thermal plasma on microbes: A review. Food Control [Internet]. 2017 May [cited 2018 Nov 20];75:83-91. Available from: https://linkinghub.elsevier.com/retrieve/pii/S0956713516307113.

16. Taghizadeh L, Brackman G, Nikiforov A, Van Der Mullen J, Leys C, Coenye T. Inactivation of biofilms using a low power atmospheric pressure argon plasma jet; the role of entrained nitrogen. Plasma Process Polym. 2015;12(1):75-81.

17. Álvarez-Pérez S, García ME, Peláez T, Blanco JL. Genotyping and antifungal susceptibility testing of multiple Malassezia pachydermatis isolates from otitis and dermatitis cases in pets: Is it really worth the effort? Med Mycol. 2016;54(1):72-9.

18. Leong C, Buttafuoco A, Glatz M, Bosshard PP. crossm Antifungal Susceptibility Testing of. J Clin Microbiol. 2017;55(6):1883-93.

19. Ziuzina D, Patil S, Cullen PJ, Keener KM, Bourke P. Atmospheric cold plasma inactivation of Escherichia coli, Salmonella enterica serovar Typhimurium and Listeria monocytogenes inoculated on fresh produce. 
DOI:10.18606/2318-1419/amazonia.sci.health.v7n3p2-11 Revista Amazônia: Science \& Health

2019, Vol. 7, № 3 .

ISSN: 2318-1419
Rodrigues ABF. Silva RA. Hage R. Khouri S.

Estudo comparativo de dois eletrodos associados ao plasma elétrico de alta frequência na redução do crescimento de cepas clínicas de Malassezia spp.

Food Microbiol [Internet]. 2014;42:109-16. Available from: http://dx.doi.org/10.1016/j.fm.2014.02.007.

20. Ziuzina D, Han L, Cullen PJ, Bourke P. Cold plasma inactivation of internalised bacteria and biofilms for Salmonella enterica serovar Typhimurium, Listeria monocytogenes and Escherichia coli. Int J Food Microbiol [Internet]. 2015;210:53-61. Available from: http://dx.doi.org/10.1016/j.ijfoodmicro.2015.05.019.

21. Annunziata M, Canullo L, Donnarumma G, Caputo P, Nastri L, Guida L. Bacterial inactivation/sterilization by argon plasma treatment on contaminated titanium implant surfaces: In vitro study. Med Oral Patol Oral Cir Bucal. 2016;21(1):e118-21.

22. Pankaj SK, Keener KM. Cold plasma: background, applications and current trends. Curr Opin Food Sci [Internet]. 2017 Aug 1 [cited 2018 Nov 20];16:49-52. Available from: https://www.sciencedirect.com/science/article/pii/S2214799316301278.

23. Lee H, Kim JE, Chung MS, Min SC. Cold plasma treatment for the microbiological safety of cabbage, lettuce, and dried figs. Food Microbiol [Internet]. 2015;51:74-80. Available from: http://dx.doi.org/10.1016/j.fm.2015.05.004. 